\title{
Health Economic Assessment of Optimal Biological Treatment for Moderate-to-Severe Psoriasis
}

\author{
Jonathan Barker ${ }^{1} \cdot$ Hannah Baker ${ }^{2} \cdot$ Ayeda Nadeem $^{2} \cdot$ Dong-Ha Gu ${ }^{3} \cdot$ Giampiero Girolomoni $^{4}(1)$
}

Accepted: 4 October 2021 / Published online: 16 October 2021

(c) The Author(s) 2021, corrected publication 2022

\begin{abstract}
Background There is limited guidance on which biologic therapies should be prioritised for the treatment of moderate-tosevere psoriasis, amongst the many available options. New mode-of-action biologics, as well as recently available biosimilars for existing biologics, continue to be developed making the choice of treatment sequence increasingly complex.

Objectives The aim of this analysis was to develop a cost-effectiveness model to determine the optimal placement of biologic therapies on the treatment pathway for psoriasis in the UK.

Methods A cohort-based Markov model was developed in Microsoft Excel, from the perspective of the National Health Service and Personal and Social Services in the UK. The model followed a hypothetical cohort of patients over a lifetime. The health states in the model were defined by Psoriasis Area and Severity Index response. In the model, patients could receive a total of four separate treatments, including three active interventions and best supportive care.

Results A fully incremental analysis was undertaken on a subset of commonly used treatment sequences. The results of the list price analyses determined the most cost-effective sequence to be adalimumab biosimilar followed by ustekinumab, secukinumab, then best supportive care. This sequence is associated with total costs of $£ 78,731$ and total quality-adjusted life-years of 14.74 over a patient's lifetime.

Conclusions This research suggests that the optimal first-line treatment in the UK is adalimumab biosimilar. The optimal second-line and third-line treatments depend on the magnitude of confidential discounts applied to the biologic treatments.
\end{abstract}

\section{Introduction}

A wide range of treatment options are available for plaque psoriasis, including biologic and non-biologic options. The choice of treatment is based on several factors including the severity of the psoriasis, patient characteristics (e.g. sex, age, comorbidities, patient's preferences) and the response to prior treatment. Additionally, clinical guidelines provide guidance in relation to the ordering of treatment. There are three major forms of conventional therapy: topical therapy, phototherapy and systemic therapy. The National Institute

Giampiero Girolomoni

giampiero.girolomoni@univr.it

1 St John's Institute of Dermatology, Faculty of Life Sciences and Medicine, King's College London, London, UK

2 York Health Economics Consortium, York, UK

3 Samsung Bioepis, Incheon, South Korea

4 Section of Dermatology, Department of Medicine, University of Verona, Verona, Italy for Health and Care Excellence (NICE) clinical guideline (CG153) on psoriasis confirms that topical therapy is the first-line treatment for people with psoriasis with phototherapy also an option when the condition is uncontrolled by topical therapy alone [1]. If these options have failed, NICE recommends that systemic options are then considered, starting with non-biologics (i.e. methotrexate and ciclosporin) and then biologic options. Therefore, systemic biological therapies are a third-line option for adults with psoriasis who have not responded to, are intolerant to, or are not eligible for standard systemic therapies or phototherapy [2].

A wide range of biological therapies are currently licensed for the treatment of plaque psoriasis in the UK and have also been formally recommended by NICE. These biologics have various modes of action, targeting as they do tumour necrosis factor (adalimumab, etanercept, certolizumab pegol, infliximab), interleukin (IL)-12/23 (ustekinumab), IL-17A (ixekizumab, secukinumab), IL-17R (brodalumab) or IL-23 (guselkumab, risankizumab, tildrakizumab) [3]. Whilst biological therapies offer superior clinical response when compared with non-biologics, this 


\section{Key Points}

A wide range of biological therapies are available for moderate-to-severe psoriasis in the UK.

However, there is little guidance on which treatments should be prioritised, which has led to variation across the country. Therefore, it is expected that sub-optimal (in terms of cost-effectiveness) sequences are being adopted in certain locations.

Adalimumab biosimilar should be considered as the firstline treatment option for the cost-effective sequence.

Adoption of adalimumab biosimilar can provide cost savings without loss of effect.

The optimal second-line and third-line treatments will depend on the level of discount applied to each biological option.

often comes with a higher cost, hence their use has historically been delayed to later in the treatment pathway. The average cost of treating a patient with moderate-to-severe psoriasis with biologics is $£ 14,255^{1}$ in the 12 months after initiation of treatment [4].

Neither NICE nor the British Association of Dermatologists (BAD) in their most recently updated guidelines [3] provides specific advice on which biologic option should be chosen by physicians. This decision is likely to be made at a local level and is based on multiple factors, including those highlighted above, as well as physician preference and purchasing agreements between manufacturers and specific National Health Service (NHS) trusts. Additionally, it is often necessary to transition a patient onto a new biologic therapy if the previous option has failed. These factors result in a high degree of variation in clinical practice.

The situation is further complicated by the advent of biosimilars. A biosimilar is a biological product that is highly similar to an approved biologic reference product, and has no clinically meaningful differences in terms of quality characteristics, biological activity, safety and efficacy based on comparability studies. Anti-tumour necrosis factor biosimilars have increased treatment options for patients and also have the potential to improve access to biologics because of the lower costs.

Both NICE and BAD have released position statements on the use of biosimilars [5, 6]. In 2017, BAD supported the use of these therapies assuming that a series of minimum reporting standards were met (e.g. robust safety monitoring

\footnotetext{
1 The average cost has been taken from a study citing 2008 costs and has been inflated to the 2019/2020 cost year, using the PSSRU 2020 NHSCII pay and prices inflation index.
}

is undertaken post-market authorisation). Similarly, the NICE position statement appears to support the use of biosimilars and, in relation to psoriasis, NICE has specifically recommended that the least expensive biosimilar option should be selected [7].

Other than this recommendation from NICE, there is limited advice from relevant bodies on which biologic therapies should be prioritised amongst the multiple options that are available. It is expected that in certain areas, sub-optimal pathways will be in operation because of less cost-effective treatment options being prioritised. The aim of this analysis was to develop a cost-effectiveness model in order to examine the optimal placement of biologic therapies, with a particular focus on biosimilars, on the treatment pathway for psoriasis in UK.

\section{Methods}

\subsection{Description of the Economic Model}

A cohort-based Markov model was developed in Microsoft Excel, from the perspective of the NHS and Personal and Social Services in the UK. The model included a hypothetical cohort of patients aged 45 years and over, of whom $50 \%$ were male. This cohort was followed over a lifetime horizon. The health states in the model were defined by the Psoriasis Area and Severity Index (PASI) score. Altogether, the following health states were included, based on the percentage improvement in PASI scores: PASI < 50, PASI 50-74, PASI 75-90, PASI $>90$. The cycle length was 14 weeks and a $3.5 \%$ discount rate was applied on costs and quality-adjusted life-years (QALYs) in accordance with NICE guidance [8].

In the model, patients could receive a total of four separate treatments, including up to three active interventions and best supportive care (BSC). Best supportive care was always the last option in a sequence. The following biologic therapies were included as possible options in the treatment sequence: reference adalimumab, biosimilar adalimumab, guselkumab, ixekizumab, risankizumab, secukinumab and ustekinumab. This is just a subset of available biologic options in the UK, chosen on the basis of common clinical practice. More information on the sequences that were adopted for the analysis are presented in the results section. The model structure is outlined in Fig. 1.

The time on each intervention was separated into two distinct phases: induction and maintenance. All treatments were assumed to have an induction period, which was used to establish whether patients responded to the treatment. Patients entered the model and received the first treatment in the sequence. At the end of the first cycle, which is the end of the induction period covering 14 weeks, patients were assigned to one of the four health states, dependent on their 


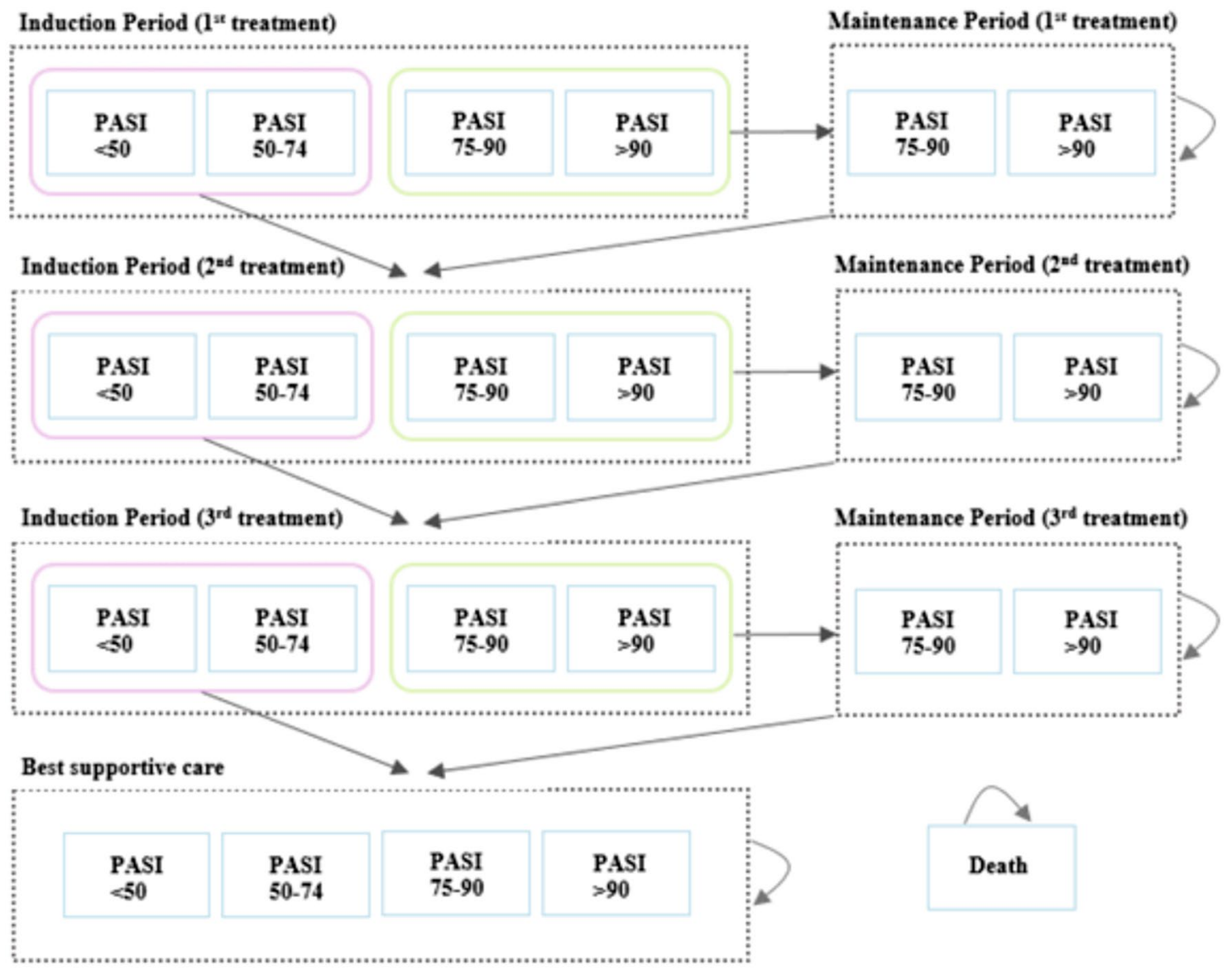

Fig. 1 Model structure. PASI Psoriasis Area and Severity Index

PASI response. A 14-week cycle length was chosen because the majority of biologic therapies have a 12- to 16 -week induction period and thus the midpoint of this range was adopted.

Patients with a PASI score improvement of $75 \%$ or greater at the end of the induction period remained on treatment and moved into the maintenance period. Therefore, it was assumed that more efficacious treatments would facilitate a higher proportion of patients remaining on treatment. During the maintenance period, patients were assumed to remain in the same health state they initially moved into (i.e. PASI 75-90 or PASI > 90). However, an ongoing risk of discontinuation was also included in the model (for example, due to loss of efficacy) and, once discontinued, patients moved onto the next treatment in the sequence.

Patients with a PASI score amelioration of less than $75 \%$ following the induction period were deemed to have an inadequate response to treatment and, therefore, they moved directly onto the second treatment in the sequence. The same process was then followed for the second treatment in the sequence and, once they discontinued from this intervention, repeated again for the third and final active treatment.
Finally, once a patient had discontinued from this third treatment they moved onto BSC, which patients were assumed to remain on until their death. Patients could enter the death state at any cycle in the model, based on age-dependent mortality rates for the general population.

It should be noted that adverse events have not been included in the analysis because biologic therapies in this indication are well tolerated and, therefore, are not a major factor on treatment choice and potential cost effectiveness. This approach also aligns with recent submissions to NICE in this indication [24-26].

\subsection{Key Model Inputs}

\subsubsection{Treatment Effectiveness}

The effectiveness of each treatment in the analysis was based on the relative change from baseline (measured at the end of the induction period) in PASI with a larger change indicating a greater response. For each biologic treatment included in the analysis, the PASI response rates were sourced from a recent Cochrane review and a network meta-analysis of 140 
psoriasis studies and were applied in the model as relative risks [9]. In some cases, PASI 50 response data for a treatment were not available, thus assumptions were made based on the data for other treatments.

The relative risks were applied to the rate of response achieved by BSC to produce an estimate of the response rate for each active treatment. The resulting efficacy estimates were assumed to remain constant for those who responded to treatment until a future discontinuation episode (i.e. a patient with a PASI $>75$ would remain in that health state during the maintenance period until they discontinued). It was also assumed that a treatment's position in the sequence did not impact on its effectiveness and thus the same PASI response rates were applied regardless of when the treatment was given. Additionally, it was assumed biosimilar therapies had equivalent response rates to their reference products. All efficacy data are presented in Table 1.

\subsubsection{Resource Use}

For patients with moderate-to-severe plaque psoriasis, resources included outpatient visits and a small number of diagnostic tests. The type and frequency of visits and tests included in the model were based upon a cost-effectiveness analysis undertaken as part of the NICE clinical guideline for psoriasis (CG153) [10]. These data are presented in Table 1.

\subsubsection{Unit Costs}

Many of the treatments included in the analysis have been recommended by NICE under the condition that they are provided at an agreed discounted price via a patient access scheme. The patient access scheme discounts are confidential and were not included in the main analyses, although discounts were approximated and applied in a scenario analysis. The unit costs of all comparators were based on list prices published by the British National Formulary [11]. A summary of unit costs for all treatments is detailed in Table 1.

The total costs per treatment for the induction and maintenance periods were estimated by multiplying the dose required for that period by the unit cost (with adjustments made to account for the total dose per unit). Monitoring costs included outpatient visits and diagnostic tests. Unit costs for all resources were obtained from NHS reference costs 2018/19 [12]. The cost of BSC was based on values adopted in CG153 [10].

\subsubsection{Health-Related Quality of Life}

Patient health-related quality-of-life scores were quantified in the model via the inclusion of utilities, which were used to estimate the total QALYs that could be expected with each sequence. Each PASI response health state was associated with a different utility in the model such that higher PASI responses were associated with greater patient quality of life. Utilities were sourced from relevant studies with moderate-to-severe psoriasis populations. These studies included NICE guidelines, clinical studies and economic evaluations [13-21]. All utilities are detailed in Table 1.

\subsubsection{Discontinuation Rates}

For patients who moved into the maintenance period for a specific intervention, there is an ongoing risk of discontinuation. The precedent in this indication was for an annual discontinuation rate of approximately $20 \%$, applied consistently across interventions. On the advice of clinical experts, the model applied different discontinuation rates for each comparator, to allow any differences between anti-tumour necrosis factors and other biologics to be demonstrated. Where discontinuation data for a treatment were not available, it was assumed to have the same discontinuation rate as another treatment of the same class. Monthly rates, with a range from 1.1 to $11.9 \%$, were converted to cycle probabilities in the model. All discontinuation rates are detailed in Table 1.

\subsubsection{Mortality}

Mortality for the general population was included in the model to capture the number of deaths, based on life tables for England and Wales [22]. The starting age in the model was 45 years and the mortality rate in the model was based on a 50:50 split of male-to-female individuals. Mortality data for the general population in England and Wales were converted from annual rates to 14 -week probabilities. No increased risk of mortality due to psoriasis or treatment was modelled as it was assumed that these would not impact mortality.

\section{Results}

There were many combinations of treatments that can be selected to form sequences in the model. It was unfeasible to include all possible permutations within this analysis. Therefore, the number of permutations was narrowed by focusing on the most commonly adopted sequences, which are described in more detail below.

It is understood adalimumab, secukinumab and ustekinumab are currently the most commonly used biologic therapies in the UK. Therefore, these three treatments made up the first analysis set. Out of six available sequences, the two sequences beginning with adalimumab were found to be the most cost-effective sequences. 
Table 1 Key model inputs

\begin{tabular}{|c|c|c|c|}
\hline Characteristic & \multicolumn{2}{|c|}{ Value } & Source \\
\hline \multicolumn{4}{|l|}{ Cohort characteristics } \\
\hline Baseline age (years) & \multicolumn{2}{|c|}{45} & [9] \\
\hline Average weight (kg) & \multicolumn{2}{|c|}{85.3} & [9] \\
\hline Proportion male & \multicolumn{2}{|c|}{$50.0 \%$} & [27] \\
\hline Level of response & \multicolumn{2}{|c|}{ Absolute response rate, $\%$} & Source \\
\hline \multicolumn{4}{|c|}{ PASI response rates for best supportive care } \\
\hline PASI 50 & \multicolumn{2}{|c|}{14.7} & [18] \\
\hline PASI 75 & \multicolumn{2}{|c|}{5.7} & [18] \\
\hline PASI 90 & \multicolumn{2}{|c|}{1.3} & [18] \\
\hline Treatment: level of response & Relative risk $(95 \% \mathrm{CI})$ & PASI response rates, $\%$ & Source \\
\hline \multicolumn{4}{|c|}{ Response by treatment (biologics) } \\
\hline Adalimumab: PASI 50 & $5.9(5.0-7.0)$ & 87.2 & {$[15]$} \\
\hline Adalimumab: PASI 75 & $9.5(7.8-11.6)$ & 54.3 & {$[9]$} \\
\hline Adalimumab: PASI 90 & $17.8(14.6-21.7)$ & 23.2 & [9] \\
\hline Guselkumab: PASI 50 & $6.6(5.3-8.0)$ & 97.7 & Assumption \\
\hline Guselkumab: PASI 75 & $11.5(9.0-14.7)$ & 65.6 & [9] \\
\hline Guselkumab: PASI 90 & $25.8(20.9-32.0)$ & 33.6 & [9] \\
\hline Ixekizumab: PASI 50 & $7.1(6.1-8.3)$ & 100.0 & {$[28]$} \\
\hline Ixekizumab: PASI 75 & $13.4(10.9-16.5)$ & 76.5 & [9] \\
\hline Ixekizumab: PASI 90 & $28.1(23.2-34.1)$ & 36.6 & [9] \\
\hline Risankizumab: PASI 50 & $6.2(4.8-8.1)$ & 91.4 & {$[29]$} \\
\hline Risankizumab: PASI 75 & $12.7(10.2-15.7)$ & 72.2 & [9] \\
\hline Risankizumab: PASI 90 & $27.7(22.9-33.5)$ & 36.0 & [9] \\
\hline Secukinumab: PASI 50 & $6.9(5.9-8.0)$ & 100.0 & {$[28]$} \\
\hline Secukinumab: PASI 75 & $13.1(10.6-16.1)$ & 74.4 & [9] \\
\hline Secukinumab: PASI 90 & $24.0(20.0-28.7)$ & 31.2 & [9] \\
\hline Ustekinumab: PASI 50 & $6.3(5.4-7.4)$ & 92.8 & {$[28]$} \\
\hline Ustekinumab: PASI 75 & $11.0(9.4-13.0)$ & 62.9 & [9] \\
\hline Ustekinumab: PASI 90 & $17.2(14.4-20.4)$ & 22.3 & [9] \\
\hline Treatment & \multicolumn{2}{|c|}{ Cycle (14 week) probability of discontinuation, $\%$} & Source \\
\hline \multicolumn{4}{|l|}{ Discontinuation (biologics) } \\
\hline Adalimumab & \multicolumn{2}{|l|}{5.1} & Calculation \\
\hline Guselkumab & \multicolumn{2}{|l|}{3.9} & Calculation \\
\hline Ixekizumab & \multicolumn{2}{|l|}{9.4} & Calculation \\
\hline Risankizumab & \multicolumn{2}{|l|}{3.9} & Calculation \\
\hline Secukinumab & \multicolumn{2}{|l|}{11.0} & Calculation \\
\hline \multirow[t]{2}{*}{ Ustekinumab } & \multicolumn{2}{|l|}{3.9} & Calculation \\
\hline & \multicolumn{2}{|c|}{ Absolute utility } & Source \\
\hline \multicolumn{4}{|l|}{ Utilities } \\
\hline Baseline & \multicolumn{2}{|l|}{0.59} & {$[13-21,30]$} \\
\hline PASI score & \multicolumn{2}{|c|}{ Incremental utility gain (from baseline) } & Source \\
\hline$<50$ & 0.05 & & {$[13-21,30]$} \\
\hline $50-74$ & 0.19 & & {$[13-21,30]$} \\
\hline $75-90$ & 0.24 & & {$[13-21,30]$} \\
\hline$>90$ & 0.28 & & {$[13-21,30]$} \\
\hline
\end{tabular}


Table 1 (continued)

\begin{tabular}{lll}
\hline Treatment & Induction cost (14 weeks), & Maintenance cost (per 14 weeks), \\
\hline Per cycle costs of treatments (at list price) & & Source \\
Adalimumab & 3168 & 2464 \\
Imraldi (adalimumab biosimilar) & 2853 & 2219 \\
Guselkumab & 6750 & 4230 \\
Ixekizumab & 9000 & 3938 \\
Risankizumab & 6652 & 3326 \\
Secukinumab & 8533 & 3929 \\
Ustekinumab & 4294 & 2512 \\
\hline Treatment & Induction cost (14 weeks) & Maintenance cost (per 14 weeks) \\
\hline Dosing regimes of treatments & & \\
Adalimumab & 7.00 & 7.00 \\
Imraldi (adalimumab biosimilar) & 7.00 & 7.00 \\
Guselkumab & 3.00 & 1.75 \\
Ixekizumab & 7.00 & 3.50 \\
Risankizumab & 2.00 & 1.17 \\
Secukinumab & 6.00 & 3.50 \\
Ustekinumab & 2.00 & 1.17 \\
\hline
\end{tabular}

Table shortened to exclude parameters related to treatments that were not included in the main analysis (i.e. etanercept, brodalumab, certolizumab, infliximab and tildrakizumab)

CI confidence interval, PASI Psoriasis Area and Severity Index

Considering that adalimumab was found to be the optimal first-line option, second and third analysis sets were then examined. Three more recent biologic therapies are ixekizumab and IL-23 inhibitors (risankizumab, guselkumab). Therefore, the second and third analysis sets focused on treatment sequences with these options alongside reference adalimumab.

The various permutations in each analysis set, and results of each permutation, are presented in Table 2. All treatments were costed at the list price (with no discounts applied). The outputs from this analysis indicate that the most costeffective sequences from the first, second and third analysis sets, respectively, are:

\section{Adalimumab $\rightarrow$ Ustekinumab $\rightarrow$ Secukinumab \\ 2. Adalimumab $\rightarrow$ Risankizumab $\rightarrow$ Ixekizumab \\ 3. Adalimumab $\rightarrow$ Ixekizumab $\rightarrow$ Guselkumab}

Within all three analysis sets presented in Table 2, the optimal sequence included reference adalimumab as the first-line option. Therefore, a fourth analysis was undertaken in which the impact of introducing biosimilar adalimumab into each of these sequences was examined. As in previous analysis sets, all treatments were costed at the list price in this analysis. The results are presented in Table 3.

The results indicate that the adoption of biosimilar adalimumab results in substantial cost savings with no loss of
Table 2 Results of the undiscounted analysis (split into three analysis sets)

\begin{tabular}{lcc}
\hline Sequence & Total costs, $£$ & Total $\mathrm{QA}$ \\
\hline First analysis set; adalimumab, & ustekinumab, secukinumab \\
$\mathrm{ADA} \rightarrow$ USTE $\rightarrow$ SECU & 81,261 & 14.74 \\
$\mathrm{ADA} \rightarrow \mathrm{SECU} \rightarrow$ USTE & 82,438 & 14.74 \\
$\mathrm{USTE} \rightarrow \mathrm{SECU} \rightarrow$ ADA & 83,769 & 14.74 \\
$\mathrm{USTE} \rightarrow$ ADA $\rightarrow$ SECU & 82,955 & 14.74 \\
$\mathrm{SECU} \rightarrow$ ADA $\rightarrow$ USTE & 89,910 & 14.74 \\
$\mathrm{SECU} \rightarrow$ USTE $\rightarrow$ ADA & 89,966 & 14.74
\end{tabular}

Second analysis set; adalimumab, ixekizumab, risankizumab

$\mathrm{ADA} \rightarrow \mathrm{IXE} \rightarrow$ RISAN $\quad 100,413-14.88$

$\mathrm{ADA} \rightarrow \mathrm{RISAN} \rightarrow \mathrm{IXE} \quad 99,727 \quad 14.88$

$\mathrm{IXE} \rightarrow \mathrm{ADA} \rightarrow \mathrm{RISAN} \quad 108,745 \quad 14.89$

$\mathrm{IXE} \rightarrow \mathrm{RISAN} \rightarrow$ ADA $\quad 109,956 \quad 14.89$

$\mathrm{RISAN} \rightarrow \mathrm{ADA} \rightarrow \mathrm{IXE} \quad 105,363 \quad 14.89$

$\mathrm{RISAN} \rightarrow$ IXE $\rightarrow$ ADA $\quad 106,313 \quad 14.89$

Third analysis set; adalimumab, ixekizumab, guselkumab

$\begin{array}{lll}\mathrm{ADA} \rightarrow \mathrm{IXE} \rightarrow \text { GUS } & 107,170 & 14.84 \\ \mathrm{ADA} \rightarrow \mathrm{GUS} \rightarrow \mathrm{IXE} & 107,459 & 14.84 \\ \mathrm{IXE} \rightarrow \mathrm{ADA} \rightarrow \text { GUS } & 115,501 & 14.84 \\ \mathrm{IXE} \rightarrow \mathrm{GUS} \rightarrow \text { ADA } & 117,777 & 14.84 \\ \mathrm{GUS} \rightarrow \mathrm{ADA} \rightarrow \mathrm{IXE} & 113,474 & 14.84 \\ \mathrm{GUS} \rightarrow \mathrm{IXE} \rightarrow \mathrm{ADA} & 114,435 & 14.84\end{array}$

$A D A$ adalimumab, GUS guselkumab, IXE ixekizumab, $Q A L Y$ qualityadjusted life-year, RISAN risankizumab, SECU secukinumab, USTE ustekinumab 
Table 3 Fully incremental results of fourth analysis set; reference adalimumab vs biosimilar adalimumab

\begin{tabular}{|c|c|c|c|}
\hline Sequence & Total costs, $£$ & Total QALYs & $\begin{array}{l}\text { Fully } \\
\text { incremental }^{\mathrm{a}} \\
\text { ICER }\end{array}$ \\
\hline $\begin{array}{l}\text { ADA BS } \rightarrow \text { USTE } \rightarrow \\
\text { SECU }\end{array}$ & 78,731 & 14.74 & - \\
\hline $\begin{array}{l}\mathrm{ADA} \rightarrow \mathrm{USTE} \rightarrow \\
\mathrm{SECU}\end{array}$ & 81,261 & 14.74 & Dominated \\
\hline $\begin{array}{l}\text { ADA BS } \rightarrow \text { RISAN } \rightarrow \\
\text { IXE }\end{array}$ & 97,196 & 14.88 & $£ 131,893$ \\
\hline $\begin{array}{l}\mathrm{ADA} \rightarrow \mathrm{RISAN} \rightarrow \\
\mathrm{IXE}\end{array}$ & 99,727 & 14.88 & Dominated \\
\hline $\begin{array}{l}\text { ADA BS } \rightarrow \text { IXE } \rightarrow \\
\text { GUS }\end{array}$ & 104,639 & 14.84 & Dominated \\
\hline $\mathrm{ADA} \rightarrow \mathrm{IXE} \rightarrow$ GUS & 107,170 & 14.84 & Dominated \\
\hline
\end{tabular}

$A D A$ adalimumab, $B S$ biosimilar, GUS guselkumab, ICER incremental cost-effectiveness ratio, IXE ixekizumab, $Q A L Y$ quality-adjusted life-year, RISAN risankizumab, SECU secukinumab, USTE ustekinumab

${ }^{a}$ In a fully incremental analysis, each option is compared with the next cheapest (non-dominated) option

effect. In a fully incremental analysis, adalimumab biosimilar $\rightarrow$ ustekinumab $\rightarrow$ secukinumab was determined to be the most cost-effective sequence. All alternative sequences but one was dominated in this analysis, meaning they were more costly but with no gain of QALYs. The only non-dominated alternative to the baseline sequence was associated with an incremental cost-effectiveness ratio of $£ 131,893$ when compared to baseline. This is substantially higher than the cost-effectiveness threshold of $£ 20,000-£ 30,000$ per QALY, which is widely accepted for UK-based costeffectiveness analyses.

However, the above analysis does not account for discounts, in the form of patient access schemes, on list prices. These discounts, whilst confidential in magnitude, were approximated and applied in a scenario analysis. In this analysis, a $40 \%$ discount was applied to the unit costs of risankizumab, ixekizumab, secukinumab and guselkumab. Reference adalimumab and ustekinumab are known to be undiscounted in the UK, thus these treatments were not discounted in the analysis. The purchase price of adalimumab biosimilar is also likely to be substantially lower than its list price. This is not because of a patient access scheme, but discounts agreed at a local level. In the scenario analysis, its price was approximated at $70 \%$ of the reference adalimumab list price. This is aligned with the approach taken in a recent similar cost-effectiveness analysis and is believed to reflect current and future market conditions [23]. The results of this analysis are presented in Table 4.

The results of the cost-effectiveness analysis with approximate discounts taken into account suggest that the optimal
Table 4 Scenario analysis; fully incremental results of the fourth analysis set with discounting on list prices

\begin{tabular}{|c|c|c|c|}
\hline Sequence & Total costs, $£$ & Total QALYs & $\begin{array}{l}\text { Fully } \\
\text { incremental }^{\mathrm{a}} \\
\text { ICER }\end{array}$ \\
\hline $\begin{array}{l}\text { ADA BS } \rightarrow \text { RISAN } \rightarrow \\
\text { IXE }\end{array}$ & 54,601 & 14.88 & - \\
\hline $\begin{array}{l}\mathrm{ADA} \text { BS } \rightarrow \text { USTE } \rightarrow \\
\text { SECU }\end{array}$ & 55,530 & 14.74 & Dominated \\
\hline $\begin{array}{l}\mathrm{ADA} \mathrm{BS} \rightarrow \mathrm{IXE} \rightarrow \\
\text { GUS }\end{array}$ & 59,035 & 14.84 & Dominated \\
\hline$\underset{\mathrm{IXE}}{\mathrm{ADA}} \rightarrow \mathrm{RISAN} \rightarrow$ & 62,236 & 14.88 & Dominated \\
\hline $\begin{array}{l}\mathrm{ADA} \rightarrow \mathrm{USTE} \rightarrow \\
\mathrm{SECU}\end{array}$ & 63,165 & 14.74 & Dominated \\
\hline $\mathrm{ADA} \rightarrow \mathrm{IXE} \rightarrow$ GUS & 66,670 & 14.84 & Dominated \\
\hline
\end{tabular}

$A D A$ adalimumab, $B S$ biosimilar, GUS guselkumab, ICER incremental cost-effectiveness ratio, IXE ixekizumab, $Q A L Y$ quality-adjusted life-year, RISAN risankizumab, SECU secukinumab, USTE ustekinumab

${ }^{\mathrm{a}}$ In a fully incremental analysis, each option is compared with the next cheapest (non-dominated) option

treatment sequence may in fact be adalimumab biosimilar $\rightarrow$ risankizumab $\rightarrow$ ixekizumab. Of course, in practice it is to be expected that different levels of discount are applied to the various treatments and thus there is substantial uncertainty surrounding this result, just as there is in the analysis using full list prices.

To check that the sequence options chosen for the final analysis set were appropriate for the discounting scenario, the analyses of the first, second and third analysis sets were also undertaken with discounts applied to the eligible treatments and this did not result in a different conclusion over the optimal sequence. The associated costs and QALYs of these analyses are not reported.

Figure 2 depicts the total costs associated with the three most cost-effective treatment sequences under the three different costing scenarios. In the first, no discounts are applied and adalimumab is costed at the reference list price. In the second, no discounts are applied and adalimumab is costed at the biosimilar list price. In the final scenario, $40 \%$ discounts are applied to all eligible reference products, and adalimumab is costed at $70 \%$ of the reference list price (as a proxy for the estimated true price of adalimumab biosimilar).

\section{Discussion}

There is currently limited guidance from both NICE and BAD on which biologic therapies should be prioritised amongst the multiple available options for people with 
Fig. 2 Total costs under different pricing scenarios. $A D A$ adalimumab, GUS guselkumab, IXE ixekizumab, RIS risankizumab, SECU secukinumab, USTE ustekinumab

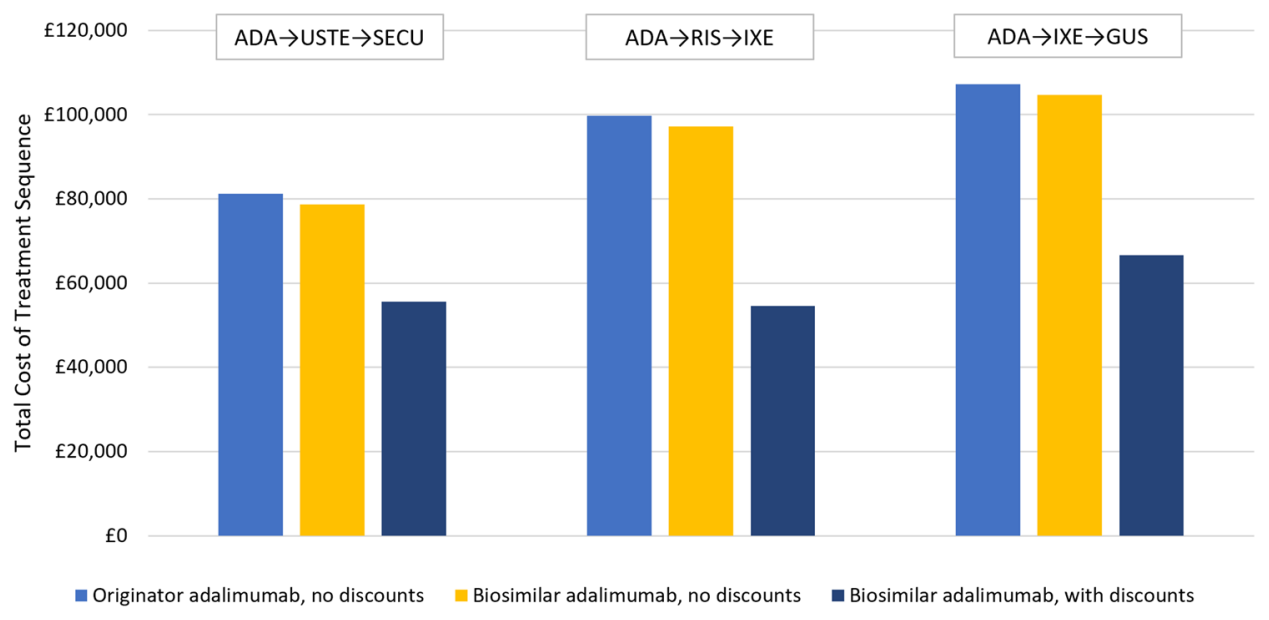

psoriasis who are eligible for these therapies. As a result, it is possible that sub-optimal pathways are in use. New modeof-action biologics as well as new biosimilars for existing biologics continue to be developed, making the choice of treatment sequence increasingly complex.

Cost-effectiveness analyses have previously been undertaken as part of the NICE technology appraisals for each emerging treatment, most recently TA596, TA574 and TA575 for risankizumab, certolizumab and tildrakizumab, respectively [24-26]. Despite this, there is no clear consensus on the most cost-effective sequence. This evaluation utilises the rich pool of comparative evidence in this area to reach a conclusion on the most cost-effective sequence of treatments. This was achieved by first establishing the optimal sequence when biosimilars are not accounted for, and then further increasing the cost effectiveness of this sequence by replacing the relevant reference product (adalimumab) with its biosimilar.

The results of the list price analyses (presented in Tables 1 and 2) suggest that the optimal sequence in Britain, of the treatments assessed, is adalimumab biosimilars $\rightarrow$ ustekinumab $\rightarrow$ secukinumab $\rightarrow$ BSC. The estimated cost saving associated with replacing adalimumab with its biosimilar as a first-line treatment in this sequence is $£ 2530$ per person treated, when treatments are costed at the list price.

However, adalimumab and ustekinumab are available at the full list price whereas all other branded treatments are known to have a commercial discount in the UK. When the commercial discounts are accounted for, it is unlikely that ustekinumab remains the optimal second-line treatment.

The true discounts on each treatment are unknown as they are confidential, and thus our analysis with a $40 \%$ discount applied to all eligible reference products, and adalimumab biosimilar costed at $70 \%$ of the reference price, was simply exploratory. However, this analysis does give an indication of what the optimal sequence might look like if similar levels of discount are assumed for all branded treatments.
If, however, there are varying levels of discount across the range of treatment options, then those with the largest discounts are likely to have a place within the optimal sequence alongside first-line adalimumab biosimilar.

Whether we favour the use of ustekinumab and secukinumab as second-line and third-line treatments, or a p19 inhibitor such as risankizumab followed by ixekizumab, it is clear that adopting a biosimilar as the first-line treatment for all eligible patients with psoriasis in the UK could result in substantial cost savings for the NHS, allowing more patients to be treated and potentially allowing earlier use of biologic treatments in the disease pathway.

The modelling approach (i.e. structure and assumptions) was largely consistent with previous models that have been submitted to NICE in this indication. Additionally, data were obtained from robust data sources wherever possible. In particular, the efficacy data utilised in this analysis were drawn from a large network meta-analysis of 140 psoriasis studies [8]. The quality of this data source is a key strength of this cost-effectiveness analysis. Unit costs were based on reputable national databases, such as the British National Formulary.

Despite the robustness of the data sources used for costs, the cost data informing the model were incomplete in the sense that many of the assessed treatments have confidential discounts applied to their list prices, and as these discounts are confidential they were not truly accounted for in the evaluation. The scenario, presented in Table 3, gives an indication of the cost-effectiveness results when a discount of $40 \%$ is applied to the list prices of all eligible reference products. In reality, it is likely that some biologics have confidential discounts larger than $40 \%$ whilst others have smaller discounts. The impact of this variation on the optimal treatment sequence can only be speculated.

Other limitations of this analysis were consistent with those commonly seen in evaluations of treatment sequences, namely the use of assumptions to fill in certain knowledge 
gaps. In particular, despite the large evidence base, it was not possible to apply differential efficacy estimates according to a treatment's place in the sequence because of a paucity of data on the impact of sequence positioning for a number of treatments. It is likely that, in reality, the efficacy of a treatment is lowered when used later in the sequence. The direction of impact of this assumption on the cost-effectiveness results is not clear. Additionally, for some treatments, the PASI 50 response data were not available in the literature and therefore assumptions were made in order to populate the model.

Only a subset of available treatment options was included in the analysis, as consideration of every possible treatment sequence is not feasible. The treatments making up this set were selected on the basis of current clinical practice and, therefore, are expected to form a relevant decision problem.

\section{Conclusions}

Results of this research suggest that the optimal firstline treatment is adalimumab biosimilar and the optimal sequence would be adalimumab biosimilar $\rightarrow$ ustekinumab $\rightarrow$ secukinumab $\rightarrow$ BSC in the UK for treating psoriasis, assuming that no discount is applied. Furthermore, adoption of adalimumab biosimilar provides $£ 2530$ of cost savings compared with using adalimumab reference product, without loss of effect. With approximate discounts accounted for, the optimal first-line treatment is still adalimumab biosimilar, and risankizumab and ixekizumab are cost-effective choices of second-line and third-line treatments, respectively. Therefore, adalimumab biosimilar should be considered as a first choice for patients with moderate-to-severe psoriasis.

\section{Declarations}

Funding Open access funding provided by Università degli Studi di Verona within the CRUI-CARE Agreement. Hannah Baker and Ayeda Nadeem are employees of York Health Economics Consortium, who were commissioned by Samsung Bioepis to provide consultancy, including developing the economic model and preparing the manuscript.

Conflicts of interest/competing interests Jonathan Barker has, in the past 5 years, attended advisory boards and/or received consultancy fees and/or spoken at sponsored symposia, and/or received grant funding from AbbVie, Almirall, Amgen, Anaptys Bio, Boehringer Ingelheim, Bristol-Meyers-Squibb, Celgene, Janssen, LEO Pharma, Lilly, Novartis, Pfizer, Samsung Bioepis, Sienna, Sun Pharma and UCB. Hannah Baker and Ayeda Nadeem work for York Health Economics Consortium, a consultancy company, which was commissioned by Samsung Bioepis to write this manuscript. Dongha $\mathrm{Gu}$ is an employee of Samsung Bioepis. Giampiero Girolomoni has been the principal investigator in clinical trials sponsored by and/or and has received personal fees from AbbVie, Abiogen, Almirall, Amgen, Biogen, Boehringer-Ingelheim, Bristol-Meyers Squibb, Eli-Lilly, Genzyme,
Leo Pharma, Merck, Novartis, OM Pharma, Pfizer, Regeneron, Samsung Bioepis and Sandoz.

Ethics approval Not applicable.

Consent to participate Not applicable.

Consent for publication Not applicable.

Availability of data and material All data generated or analysed during this study are included in this published article and its supplementary information files.

Code availability Not applicable.

Author contributions Model conceptualisation: all authors; data acquisition: $\mathrm{HB}, \mathrm{AN}$; data analysis and interpretation: $\mathrm{HB}, \mathrm{AN}$; manuscript preparation: $\mathrm{HB}, \mathrm{AN}, \mathrm{DG}$; manuscript editing: all authors; manuscript review: all authors.

Open Access This article is licensed under a Creative Commons Attribution-NonCommercial 4.0 International License, which permits any non-commercial use, sharing, adaptation, distribution and reproduction in any medium or format, as long as you give appropriate credit to the original author(s) and the source, provide a link to the Creative Commons licence, and indicate if changes were made. The images or other third party material in this article are included in the article's Creative Commons licence, unless indicated otherwise in a credit line to the material. If material is not included in the article's Creative Commons licence and your intended use is not permitted by statutory regulation or exceeds the permitted use, you will need to obtain permission directly from the copyright holder. To view a copy of this licence, visit http://creativecommons.org/licenses/by-nc/4.0/.

\section{References}

1. National Institute for Health and Care Excellence. Psoriasis: assessment and management [CG153]. https://www.nice.org.uk/ guidance/cg153. Accessed 13 Oct 2021.

2. National Institute for Health and Care Excellence. Pathway for psoriasis. https://pathways.nice.org.uk/pathways/psoriasis. Accessed 11 Oct 2021.

3. Smith CH, Yiu ZZN, Bale T, Burden AD, Coates LC, Edwards W, et al. British Association of Dermatologists guidelines for biologic therapy for psoriasis 2020: a rapid update. Br J Dermatol. 2020;183(4):628-37.

4. Fonia A, Jackson K, LeReun C, Grant DM, Barker JNWN, Smith $\mathrm{CH}$. A retrospective cohort study of the impact of biologic therapy initiation on medical resource use and costs in patients with moderate to severe psoriasis. Br J Dermatol. 2010;163(4):807-16.

5. British Association of Dermatologists. British Association of Dermatologists' position Statement on biosimilars. https://www.bad. org.uk/healthcare-professionals/badbir. Accessed 13 Oct 2021.

6. National Institute for Health and Care Excellence. NICE's biosimilars position statement. https://www.nice.org.uk/Media/Default/ About/what-we-do/NICE-guidance/NICE-technology-appraisals/ biosimilars-statement.pdf. Accessed 13 Oct 2021.

7. National Institute for Health and Care Excellence. NICE pathway: systemic biological therapy for psoriasis. https://pathways.nice. org.uk/pathways/psoriasis. Accessed 13 Oct 2021.

8. National Institute for Health and Care Excellence. Guide to the methods of technology appraisal 2013 [PMG9]. https://www.nice. org.uk/process/pmg9. Accessed 13 Oct 2021. 
9. Sbidian E, Chaimani A, Afach S, Doney L, Dressler C, Hua C, et al. Systemic pharmacological treatments for chronic plaque psoriasis: a network meta-analysis. Cochrane Database Syst Rev. 2020;1(1): CD011535.

10. National Institute for Health and Care Excellence. Clinical guideline 153: psoriasis: assessment and management. London: National Institute for Health and Care Excellence; 2012.

11. National Institute for Health and Care Excellence. British National Formulary. https://bnf.nice.org.uk/. Accessed 11 Oct 2021.

12. National Health Service. National cost collection 2018/19. https:// www.england.nhs.uk/national-cost-collection/. Accessed 11 Oct 2021.

13. National Institute for Health and Care Excellence. Etanercept and efalizumab for the treatment of adults with psoriasis TA103. https://www.nice.org.uk/guidance/ta103. Accessed 13 Oct 2021.

14. National Institute for Health and Care Excellence. Infliximab for the treatment of adults with psoriasis TA134. https://www.nice. org.uk/guidance/ta134. Accessed 13 Oct 2021.

15. National Institute for Health and Care Excellence. Adalimumab for the treatment of adults with psoriasis TA146. https://www. nice.org.uk/guidance/ta146. Accessed 13 Oct 2021.

16. National Institute for Health and Care Excellence. Ustekinumab for the treatment of adults with moderate to severe psoriasis TA180. https://www.nice.org.uk/guidance/ta180. Accessed 13 Oct 2021.

17. National Institute for Health and Care Excellence. Ixekizumab for treating moderate to severe plaque psoriasis TA442. https://www. nice.org.uk/guidance/ta442. Accessed 13 Oct 2021.

18. National Institute for Health and Care Excellence. Brodalumab for the treatment of adults with moderate to severe psoriasis TA511. https://www.nice.org.uk/guidance/ta511. Accessed 13 Oct 2021.

19. Shikiar R, Willian MK, Okun MM, Thompson CS, Revicki DA. The validity and responsiveness of three quality of life measures in the assessment of psoriasis patients: results of a phase II study. Health Qual Life Outcomes. 2006;4(1):71.

20. Pickard AS, Gooderham M, Hartz S, Nicolay C. EQ-5D health utilities: exploring ways to improve upon responsiveness in psoriasis. J Med Econ. 2017;20(1):19-27.
21. Sizto S, Bansback N, Feldman SR, Willian MK, Anis AH. Economic evaluation of systemic therapies for moderate to severe psoriasis. Br J Dermatol. 2009;160(6):1264-72.

22. Office for National Statistics. National life tables: England and Wales 2016-18. https://www.ons.gov.uk/peoplepopulationandc ommunity/birthsdeathsandmarriages/lifeexpectancies/bulletins/ nationallifetablesunitedkingdom/2016to2018. Accessed 13 Oct 2021.

23. Weber TBM, Pemberton-Ross P, Haines P, Bas C, Pieper B, Ebbers $\mathrm{H}$, et al. PBI10 cost effectiveness analysis for the treatment of psoriasis: the use of adalimumab biosimilars. Value Health. 2020;23:S411-2.

24. National Institute for Health and Care Excellence. Risankizumab for treating moderate to severe plaque psoriasis [TA596]. https:// www.nice.org.uk/guidance/ta596. Accessed 13 Oct 2021.

25. National Institute for Health and Care Excellence. Certolizumab pegol for treating moderate to severe plaque psoriasis [TA574]. https://www.nice.org.uk/guidance/ta574. Accessed 13 Oct 2021.

26. National Institute for Health and Care Excellence. Tildrakizumab for treating moderate to severe plaque psoriasis [TA575]. https:// www.nice.org.uk/guidance/ta575. Accessed 13 Oct 2021.

27. Gelfand JM, Weinstein R, Porter SB, Neimann AL, Berlin JA, Margolis DJ. Prevalence and treatment of psoriasis in the United Kingdom: a population-based study. Arch Dermatol. 2005;141(12):1537-41.

28. Sawyer L, Fotheringham I, Wright E, Yasmeen N, Gibbons C, Holmen MA. The comparative efficacy of brodalumab in patients with moderate-to-severe psoriasis: a systematic literature review and network meta-analysis. J Dermatol Treat. 2018;29(6):557-68.

29. Clinical Review Report: Risankizumab (Skyrizi): (AbbVie): indication: for the treatment of adult patients with moderate to severe plaque psoriasis who are candidates for systemic therapy or phototherapy. https://cadth.ca/risankizumab. Accessed 13 Oct 2021.

30. Anis AH, Bansback N, Sizto S, Gupta SR, Willian MK, Feldman $\mathrm{S}$. Economic evaluation of biologic therapies for the treatment of moderate to severe psoriasis in the United States. J Dermatol Treat. 2011;22(2):65-74. 